\title{
Hybrid Detectors for High Resolution Imaging.
}

$\underline{\text { A. Bergamaschi }}{ }^{1, *}$, M. Andrä ${ }^{1}$, R. Barten ${ }^{1}$, M. Brückner ${ }^{1}$, S. Chiriotti ${ }^{1}$, C. David ${ }^{1}$, R. Dinapoli ${ }^{1}$, E. Fröjdh $^{1}$, D. Greiffenberg ${ }^{1}$, M. Lebugle ${ }^{1}$, C. Lopez-Cuenca ${ }^{1}$, D. Mezza ${ }^{1}$, A. Mozzanica ${ }^{1}$, M. Ramilli ${ }^{1}$, S. Redford $^{1}$, C. Ruder ${ }^{1}$, B. Schmitt ${ }^{1}$, X. Shi ${ }^{1}$, D. Thattil ${ }^{1}$, G. Tinti ${ }^{1}$, S. Vetter ${ }^{1}$, J. Zhang ${ }^{1}$

${ }^{1 .}$ Paul Scherrer Institut, Villigen PSI, Switzerland.

* Corresponding author, anna.bergamaschi@psi.ch

In the last 15 years, hybrid detectors have become very common in various X-ray applications as a result of the improvement of their yield and consequent cost reduction [1]. The room temperature operation of hybrid detectors allows simple experimental setups, high reliability and ease of use. Detectors can be tiled to cover large areas, with a frame rate up to a few thousand frames per second for millions of pixels. However, the need of bump-bonding to interconnect the sensor absorbing the radiation with the read-out electronics, puts a limit on the minimum pixel size, and consequently on the spatial resolution that can be achieved.

For this reason, in the case of high resolution imaging applications, indirect conversion detectors are normally used, thanks to the possibility to exploit optical magnification between the phosphor screen converting X-rays into visible light and the monolithic detector (usually a CCD or a CMOS sensor) in order to achieve sub-micron spatial resolution [2]. However, the Detective Quantum Efficiency (DQE) of the detector, defined as the signal-to-noise ratio (SNR) squared relative to an ideal detector, can be significantly reduced compared to the absorption efficiency of the phosphor screen, mainly because of the inefficiency of the light conversion and collection processes. Moreover, the spatial resolution is often limited by light diffusion in the phosphor screen and by the optical processes rather than by the pixel size of the detector.

On the other hand, the direct conversion of X-rays into electric charge in the sensor and the low noise offered by hybrid detectors allows single photon sensitivity in the hard X-ray energy range. This means that the SNR of the image is purely determined by the Poisson statistic on the number of detected photons and the DQE equals the absorption efficiency of the sensor. A $300 \mu \mathrm{m}$ thick silicon sensor is standard for many applications, but thicker silicon sensors up to $1 \mathrm{~mm}$ or alternative high-Z semiconductor materials (e.g. CdTe, GaAs) can also be used to improve the quantum efficiency for hard X-rays.

The MÖNCH detector is being developed at the Paul Scherrer Institut in order to explore the spatial resolution limits of direct conversion hybrid detectors. It provides a unique $25 \mu \mathrm{m}$ pixel pitch together with an outstanding low noise of less than $150 \mathrm{eV}$ r.m.s., allowing to detect $12 \mathrm{keV}$ photons with a SNR higher than 80 [3]. Prototypes up to $400 \times 400$ pixels $\left(1 \times 1 \mathrm{~cm}^{2}\right)$ have been successfully bump bonded and characterized [4].

The diffusion of the charge generated by the X-rays during the charge collection process inside the silicon sensor, which is of the order of $20 \mu \mathrm{m}$, would limit the spatial resolution of the detector for smaller pixels, which would also be at the limit for the optical lithography involved in the sensor fabrication, the bump bonding process and design of the readout electronics. Nevertheless, it has been shown that by detecting the sharing of the charge cloud between neighboring pixels, a spatial resolution 
better than the pixel pitch can be achieved by using interpolation algorithms [5,6]. As an example, figure 1 shows the X-ray image of a section of a Siemens Star with pitches ranging between 30 and $0.5 \mu \mathrm{m}$ fabricated at PSI in gold on a silicon substrate acquired at $15 \mathrm{keV}$ at the SYRMEP beamline at Elettra [8] before and after interpolation to $1 \mu \mathrm{m}$ bins, resulting in $25 \times 25$ virtual sub-pixels per physical pixel. For comparison, in the case of the non interpolated image, the photons of a single pixel have been uniformly redistributed over the $25 \times 25$ bins. No structure can be distinguished before interpolation, while pitches down to about $6 \mu \mathrm{m}$, i.e. much smaller than the physical pixel size of the detector, can be resolved after interpolation.

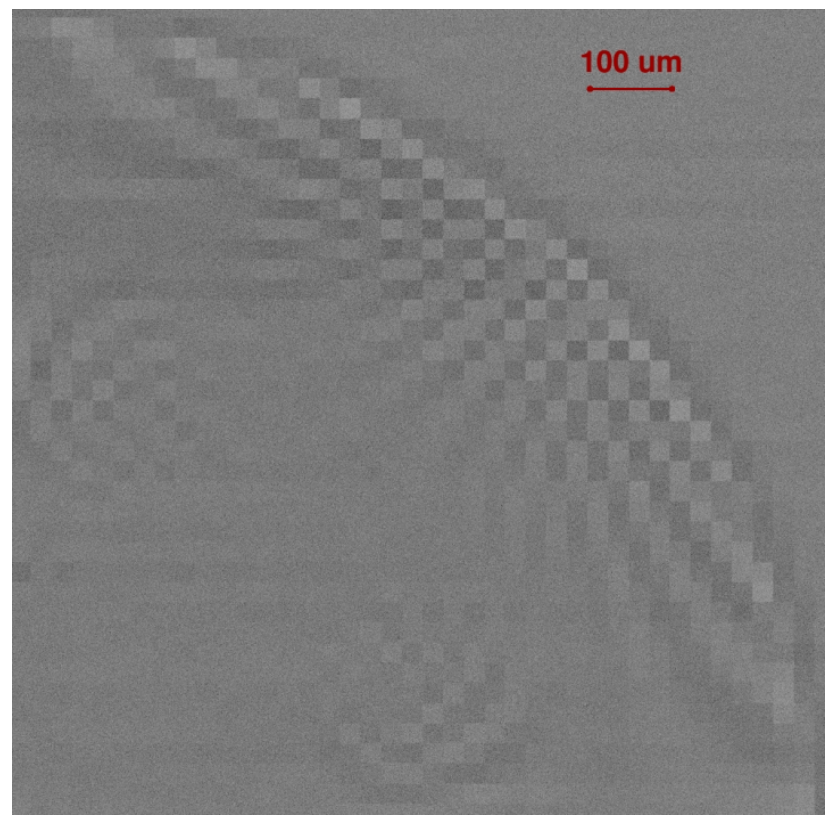

(a)

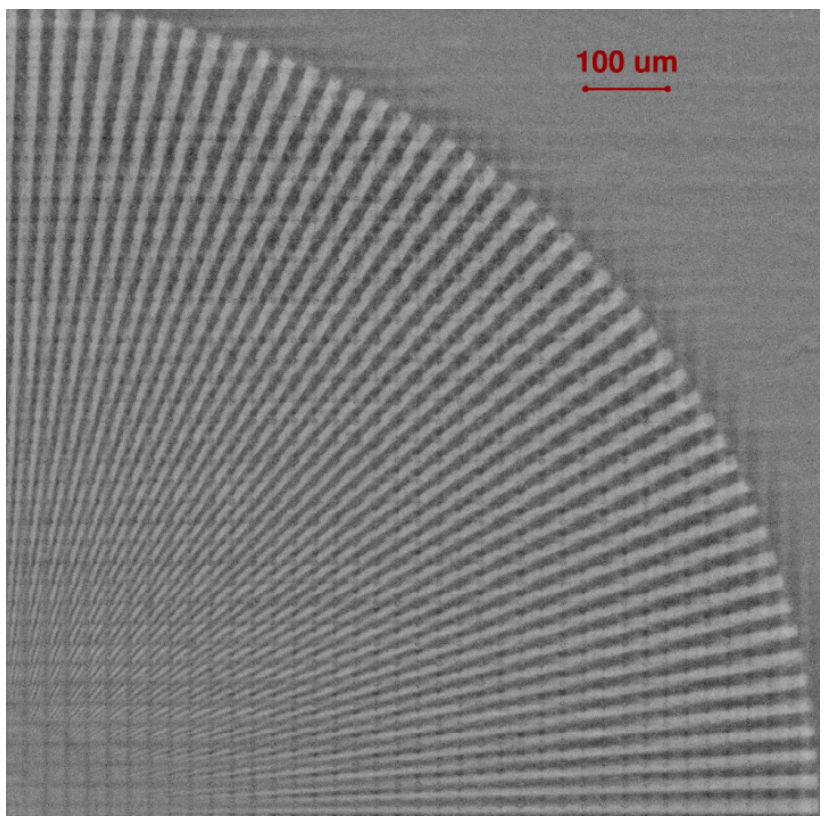

(b)

Figure. 1. X-ray image of a Siemens Star fabricated at PSI in gold on a silicon substrate with a pitch varying between 30 and $0.5 \mu \mathrm{m}$ acquired at $15 \mathrm{keV}$ at the SYRMEP beamline at Elettra [8] a) before and b) after interpolation to $1 \mu \mathrm{m}$ bins.

Position interpolation has the advantage of limiting the number of physical pixels of the detector simplifying its design. However, the technique is still under development and is very sensitive to small changes in the experimental conditions (e.g. photon flux) and the interpolated images often present artefacts due to non uniform redistribution of photons. The spatial resolution can be improved by operating the detector at lower bias voltage or by using thicker sensors in order to enhance the charge sharing. However, the spatial resolution is intrinsically not uniform within the pixel (higher in the corners, lower in the center due to reduced charge sharing), which can be disturbing for some imaging techniques.

The main disadvantage is that interpolation can work only for isolated photons i.e. only at low photon fluxes when less than a photon is detected in a 3x3 pixel cluster. Currently, with a maximum frame rate of $3 \mathrm{kfps}$, the spatial resolution of $\mathrm{MÖNCH}$ can be improved better than the pixel pitch up to a maximum photon flux of 5E5 $\mathrm{ph} / \mathrm{s} / \mathrm{mm}^{2}$. Although dose effective, this low flux involves long exposure times and does not allow for fast or time resolved imaging. However, it can well match the requirements of X-ray microscopes, where the performance of monolithic detectors suffers from long exposure times and poor DQE. The detector can still be used at higher fluxes, but in this case the spatial resolution is 
limited by the pixel pitch and cannot be improved by interpolation algorithms [7]. An improvement in the maximum photon flux supported by interpolation can only come by a faster frame rate. However, although the data transfer rate could eventually be increased and the data output could be further parallelized to speed up the readout, these improvements are in contrast with the requirements of detectors with larger field of view, which would also increase the number of pixels to be readout. In the next few years we plan to design larger MÖNCH detector systems to cover up to $4 \times 3 \mathrm{~cm}^{2}$ i.e. almost 2 Million pixels. This large field of view will be beneficial to many imaging applications, but brings major challenges in terms of electronic design, bump-bond yield and data acquisition backend.

Currently, interpolation is performed offline and data processing is still 5-10 times slower than the acquisition, depending on the computing platform. Therefore, a large data amount (ca. 1TB/hour) has to be stored and processed in order to obtain the interpolated image. Often on-the-fly interpolation is performed either at slower frame rates or on a partial dataset for image preview, while the whole data are processed offline. We are exploring possibilities to speed up the data processing by optimization of the code or by using GPUs.

An analog detector like MÖNCH can be particularly useful with microfocus X-ray tubes, where its energy resolving power can be exploited and the flux is often low enough for separating single photons. Color imaging with an energy resolution better than $400 \mathrm{eV}$ FWHM can be performed. In this case, the images must be separated into different energy windows in order to apply interpolation, since charge sharing strongly depends on the X-ray energy. This makes interpolation even more challenging, but can bring improvements in many applications, e.g. X-ray grating interferometry.

Thick silicon sensors and high-Z sensor materials are under investigation in order to increase the quantum efficiency of the detector for hard X-rays. Position interpolation using MÖNCH has been demonstrated down to $2 \mathrm{keV}$ [4], but we are trying to further reduce the electronic noise in order to operate also in the soft X-ray energy range. In this case, the quantum efficiency of the detector has to be improved by reducing the thickness of the entrance window of the detector. Additionally, we plan to extend the dynamic range of the detector by using dynamic gain switching [8] in order to perform experiments at FELs as well as at synchrotrons, not necessarily with interpolation.

Considering the huge advancement of hybrid detectors in the last decade, we expect that the promising results obtained so far using interpolation will find application in many imaging experiments, combining the advantages of direct conversion detectors with high spatial resolution down to the micron level.

\section{References:}

[1] T. Hatsui and H. Graafsma, IUCrJ 2 (2015) p. 371.

[2] J. C. Larsson et al, Med. Phys. 43 (2016) p. 2731 T.

[3] R. Dinapoli et al, J. Instrum. 9 (2014) p. C050115.

[4] M. Ramilli et al, J. Instrum. 12 (2017) p. C01071.

[5] S. Cartier, M. Kagias et al, J. Synch. Rad. 23 (2016) p. 1462.

[6] S. Ihle et al, J. Instrum. 12 (2017) p. P02005.

[7] C. Dullin et al, J. Synch. Rad. 25 (2018) p. 565.

[8] B. Henrich et al, Nucl. Instr. Meth. A 633 (2011) p. S11. 
https://doi.org/10.1017/S1431927618013910 Published online by Cambridge University Press 